\title{
Adaptation of the INTERGROWTH-21st Neurodevelopment Assessment (INTER-NDA) to the Context of the English- speaking Caribbean
}

\section{Randall Waechter}

St. George's University, School of Medicine

\section{Roberta Evans}

The Windward Islands of Research and Education Foundation

\section{Sean Hanna ( $\sim$ seanhanna18@gmail.com )}

St. George's University https://orcid.org/0000-0002-0086-9641

\section{Toni Murray}

The Windward Islands Research and Education Foundation

\section{Cassandra Mobley}

The Windward Islands Research and Education Foundation

\section{Stephanie Holmes}

The Windward Islands Research and Education Foundation

\section{Rashida Isaac}

The Windward Islands Research and Education Foundation

\section{Rebeca Wolfe}

The Windward Islands Research and Education Foundation

\section{Elbernezer Andrew}

The Windward Islands Research and Education Foundation

\section{Barbara Landon}

Psychological Services Center, St. George's University

\section{Michelle Fernandes}

National Institute of Health Research Biomedical Research Center \& Faculty of Medicine

\section{Research article}

Keywords: INTER-NDA, neurodevelopmental assessment, early childhood development, child assessment, assessment translation

Posted Date: June 10th, 2021

DOI: https://doi.org/10.21203/rs.3.rs-594940/v1

License: (c) (i) This work is licensed under a Creative Commons Attribution 4.0 International License. Read Full License

Version of Record: A version of this preprint was published at BMC Pediatrics on January 4th, 2022. See the published version at https://doi.org/10.1186/s12887-021-03039-7. 


\section{Abstract}

Adaptation of standardized early child development (ECD) assessments to low- and middle-income countries can be challenging because of culture specific factors relating to language, content, context, and tool administration, and because the reliance of these tests on specialist healthcare professionals limits their scalability in low resource settings. We report the cross-cultural adaptation of an international, standardized ECD instrument, the INTERGROWTH-21st Project Neurodevelopment Assessment (INTER-NDA), measuring cognitive, language, motor and behavioural outcomes in 2-year-olds, from a UK-based English-speaking population to the English-speaking Caribbean. Children aged 20-30 months were recruited from a pre-existing randomized controlled neurodevelopment intervention study in Grenada, West Indies. Eight of 37 INTER-NDA items (22\%) were culturally and linguistically adapted for implementation in the Caribbean context. Protocol adherence across seven newly-trained non-specialist child development assessors was $89.9 \%$; six of the seven assessors scored $\geq 80 \%$. Agreement between the expert assessor and the non-specialist child development assessors was substantial ( $\mathrm{K}=0.89$ to 1.00 (95\% $\mathrm{Cl}[0.58,1.00])$. The inter-rater and test-retest reliability for non-specialist child development assessors was between $\mathrm{K}=0.99-1.00(95 \% \mathrm{Cl}[0.98,0.99])$ and $\mathrm{K}=0.76-1.00(95 \% \mathrm{Cl}$ $[0.33,1.00])$ across all INTER-NDA domains.

\section{Introduction}

An estimated 249.4 million children under the age of five years, in low and middle-income countries (LMICs), are at risk of failing to achieve their full neurodevelopmental potential (Grantham-McGregor et al., 2007; Lu, Black, \& Richter, 2016). The higher incidence and prevalence of neurodevelopmental impairment in LMICs than in high-income countries is well evidenced (Durkin, 2002; Grantham-McGregor et al., 2007; Lu, Black, \& Richter, 2016). Accurate measurement of children at risk of neurodevelopmental impairment (NDI) is key to targeting interventions to those at highest risk, especially in LMIC settings where healthcare resources are often limited (Fernandes et al., 2020). Moreover, quantification of NDI burden in and across populations is the first important step in measuring its long-term impact and evaluating the effectiveness of intervention strategies. Nevertheless, epidemiological data on normative child development are sparse or non-existent in many parts of the world, particularly in those areas where children are most at risk (Black \& Lawn, 2018). This creates challenges for early child development (ECD) surveillance in LMICs, where ECD outcomes in children from disparate geographical and/or cultural contexts are evaluated using instruments that have not been subjected to a rigorous standardization, adaptation and cultural-customization process for use in these settings. This is particularly significant in ECD assessments because social, cultural and language-related factors can adversely affect a child's understanding of a test item and his/her subsequent performance on the ECD measure (Semrud-Clikeman et al., 2016). The use of inadequately adapted ECD assessment tools, at a population-health level, could result in over- or underestimation of the prevalence of NDIs and, at an individual level, result in the misclassification of children as being at risk (or not) of NDIs. The life-course and public health consequences of either outcome are significant.

The importance of cross-cultural adaptation of ECD instruments, their adequate translation in the context of colloquialisms and accents; and the robust evaluation of adapted instruments has been highlighted in the World Bank's Toolkit for the assessment of ECD in children aged under five years in LMICs (Fernald, Kariger, Engle, \& Raikes, 2009). The Toolkit encourages cross-cultural adaptations because the data from the ECD assessments can provide tremendous developmental insight in areas where formal testing is unavailable, or rarely practiced. Measures that are not amended tend to demonstrate biases and poorer performance levels because some items do not function in the same manner across cultures (Fernald et al., 2009). Moreover, the interpretation of unfamiliar phraseology and its linkage to familiar lexical items to express roughly the same concept, are executive functions that can only be expected to emerge during mid to late childhood (Farrar \& Ashwell, 2012). It is widely acknowledged that sociolinguistics is critically important when administering a standardized assessment within a culture, including within the same language, as pronunciation, tonality, sentence placement and the words themselves are very important in language processing (Gladkova, 2012). 
One region in which there is limited data on ECD is the 700-island Caribbean basin with a regional population of 44 million (United Nations, Department of Economic and Social Affairs, Population Division, 2017). Of the 33 countries and individual territories in the region, ten of these are classified as LMICs (World Bank, 2018). Previously, tropical infections represented the greatest disease burden in the region, however in recent years the burden has shifted to chronic diseases (during adulthood) and ECD disorders, including NDIs (during childhood) (Razzouk et al., 2008). Importantly, the few coordinated efforts to assess individual or groups of young children in the Latin American and Caribbean region suggest that approximately $15 \%$ of children are at high risk of NDIs (Lu, et al., 2016). Despite this, there are, to our knowledge, no ECD tools adapted to and standardized in the cultural and linguistic context of the Englishspeaking Caribbean. We hypothesize that culturally-adapted ECD assessments specific to the Caribbean do not exist because the region's residents speak dialects of English, French or Spanish, and therefore European and American instruments have been directly exported there without a consideration of how linguistic and cultural differences between these disparate geographical and social populations might influence the test delivery and children's performance.

The current study addresses this methodological gap in ECD measurement in the Caribbean by, for the first time, (1) adapting (from British to Caribbean English) and culturally customizing a rapid, multi-dimensional, international, standardized ECD instrument, The INTERGROWTH-21st Neurodevelopment Assessment (INTER-NDA), for the measurement of cognitive, motor, language and behavioral outcomes in young Caribbean children, (2) comparing the psychometric properties of the adapted to the original tool and (3) evaluating the ability of non-specialist child development assessors to administer and score the adapted version of the INTER-NDA.

\section{Methods}

\section{Study Location}

The study was carried out in the tri-island nation of Grenada, West Indies. Grenada has been an independent Englishspeaking nation since 1974. Thirty-two percent of Grenada's population is under 14 years of age (Government of Grenada, 2005). Throughout the region, approximately half of all households are headed by a single parent (Bakker, Elings-Pels, \& Reis, 2009). Despite its ranking as an upper middle-income country, approximately a third of its 112,000 residents live in poverty. Unemployment is reported at $20 \%$ and the nation's debt ratio of $110 \%$ GDP ranks second worldwide (World Bank, 2016). The gross national income per capita was reported at $\$ 11,650$ United States Dollars in 2014 (World Bank, 2016). Ethnically, more than $80 \%$ of the population identifies as Afro-Caribbean; the remainders are of mixed, or East Indian ancestry (Central Intelligence Agency, 2018).

\section{Sample - Child Development Assessors}

Table 1 describes the characteristics of the seven non-specialist child development assessors. All of the assessors were females and native Caribbean-English speakers. Five of the assessors were Caribbean nationals and two were American citizens. None of these assessors were psychologists or healthcare professionals, and none had a formal education beyond an undergraduate degree. Their income, representative of their socioeconomic status, was equivalent to Grenada's 2014 gross national income per capita at $\$ 11,750$ United States Dollars (World Bank, 2016), adjusted for inflation.

Table 1 Characteristics of 7 non-specialist child developmental assessors 


\begin{tabular}{|ll|}
\hline Characteristic & $\boldsymbol{N}(\%)$ or Mean (SD) \\
\hline Age & $29.8(10.8)$ \\
\hline Sex (Female) & $7(100 \%)$ \\
\hline Number of years of Education & $22.3(3.1)$ \\
\hline Native language (Caribbean-English) & $7(100 \%)$ \\
\hline Ethnicity & Caribbean - $5(71.43 \%)$ \\
& American $-2(28.57 \%)$ \\
\hline
\end{tabular}

\section{Sample - Child Participants}

Each of the seven non-specialist child developmental assessors assessed three children, for a total of 21 child participants. Children ranged from 20 to 30 months old and were recruited from the "Savings Brains Grenada" (SBG) project - a randomized controlled trial study assessing the impact of a Conscious Discipline intervention on reducing corporal punishment rates and improving neurodevelopmental outcomes among Grenadian children (Landon \& Waechter, 2016). The children included in this report were recruited from four day-care centers around the capital city of St. George's. The sample characteristics of the participating children are presented in Table 2.

Table 2

\begin{tabular}{|ll|}
\hline Characteristic & $\mathbf{N}(\%)$ or Mean (SD) \\
\hline Age & $29.8(10.8)$ \\
\hline Sex (Female) & $7(100 \%)$ \\
\hline Number of years of Education & $22.3(3.1)$ \\
\hline Native language (Caribbean-English) & $7(100 \%)$ \\
\hline Ethnicity & Caribbean $-5(71.43 \%)$ \\
& American $-2(28.57 \%)$ \\
\hline \multicolumn{2}{|l|}{ Characteristics of 21 Child Participants } \\
\hline
\end{tabular}

\section{Measures}

\section{The INTERGROWTH-21st Project Neurodevelopment Assessment (INTER-NDA):}

The INTER-NDA is a comprehensive, rapid assessment of cognition, (fine and gross) motor skills, language and (positive and negative) behavior for children aged 22-30 months (Appendix A) (Fernandes et al., 2020). Its 37 items are administered using a combination of psychometric techniques (direct administration, concurrent observation and caregiver reports) in approximately 15 minutes. Children's performance on the INTER-NDA is scored across a spectrum of abilities, rather than on a predefined checklist and, therefore, affords a wider description of a child's faculties. It has been reported to have strong agreement with the Bayley Scales of Infant and Toddler Development III edition, (BSID-III) (interclass correlation coefficients $0.75-0.88, p<0.001$ for all domains with little to no bias on Bland Altman analysis); satisfactory internal consistency (Cronbach's alpha 0.56-0.81) and good unidimensionality across subscales (Comparative Fit Index $=0.90$; Tucker-Lewis Index $=0.94$ ) and good levels of inter-rater $(\mathrm{k}=0.70 ; 95 \% \mathrm{Cl} 0.47$ to 0.88$)$ and test-retest reliability ( $k=0.79 ; 95 \% \mathrm{Cl} 0.48$ to 0.96$)$ (Fernandes et al., 2014; Murray et al., 2018). 
The INTER-NDA is designed for use across socioeconomic groups and populations. Its operation manual, standardization protocol and forms are freely available at www.intergrowth21.org.uk. The kit consists of common household items encountered across the world. The INTER-NDA was developed in 2014 by the International Fetal and Newborn Growth Consortium for the 21st Century (INTERGROWTH-21st) Project, a population-based, longitudinal study in five countries, including Brazil, India, Italy, Kenya, and the United Kingdom (Villar et al., 2013). In all INTERGROWTH21st Project study sites, the INTER-NDA was translated into the local languages of the sites using the WHO Mental Health Initiative translation guidelines, which included processes of cultural customization, translation and back translation (Fernandes et al., 2020). The INTER-NDA's norms are international standards (rather than population-specific references) for child development at 2 years of age, constructed from the INTERGROWTH-21st Project population using, the WHO's prescriptive methodology. Scaled INTER-NDA domain scores are interpreted against the INTER-NDA's standards to ascertain a child's risk of no (> 10th centile), mild-to-moderate (3rd to 10th centile) or severe (<3rd centile) neurodevelopmental delay for each of the domains (Fernandes et al., 2020).

\section{Training of Child Development Assessors}

Seven non-specialist child developmental assessors were trained in the INTER-NDA in Grenada over a five-day period by the developer of the INTER-NDA (MF) who is a UK-based paediatrician, and who served as the expert assessor (expert) for the purpose of this study. During the training process, importance was paid to the conceptual basis of each item, the accurate administration of tasks to the child, as well as the accurate and objective reporting of the child's performance on each item. All sessions were video recorded, and the expert observed the trainee-assessors carrying out three assessments each on 20-30-month-old children. After these sessions, the expert provided each trainee-assessor with feedback on their administration and scoring of the INTER-NDA and on their interaction with the child and the caregiver.

\section{INTER-NDA adaptation process to the Caribbean context}

The process of the linguistic and cultural adaptation of the INTER-NDA was undertaken on day three of the training, after each trainee-assessor had assessed at least one child, and involved all trainee-assessors, the study PIs and the expert assessor. This process adhered to the previously published, recommended guidelines for the adaptation of an ECD instrument (Fernald et al., 2009). The process consisted of the following steps:

1. The trainee-assessors and PIs evaluated each of the 37 INTER-NDA items for linguistic and cultural relevance. Items considered to benefit from amendments to fit the local context were identified.

2. The trainee-assessors proposed alternatives to the phraseology of the items identified above. These options were discussed with the study PIs and expert assessor. Each option was scrutinized for contextual relevance by the trainee and expert assessors; any conflicts identified were discussed between all the assessors and alternative phraseology was proposed.

3. The eight adapted items were compared to the original INTER-NDA items by the expert assessor to confirm conceptual equivalence in accurately screening for NDIs. Conflicts identified were discussed with the trainee-assessors, alternative phraseology was considered and the process of assessment for contextual and conceptual equivalence repeated until a consensus was achieved.

4. The final list of adapted items were included into the measure and piloted on children aged 20-30 months.

\section{Evaluation of the INTER-NDA's administration and scoring by non- specialist child development assessors}

Each non-specialist child developmental assessor was evaluated for their ability to (1) administer and (2) score the INTER-NDA in a standardized manner according the INTERGROWTH-21st Project protocols. To assess their ability to 
administer the INTER-NDA in a standardized manner, each non-specialist child development assessor was rated for protocol adherence on the INTERGROWTH-21st Project's INTER-NDA protocol adherence checklist (Appendix B; http://www.medscinet.net/Intergrowth/patientinfodocs/Standardisation\%20Protocol.pdf) by the six other non-specialist child developmental assessors and the expert assessor. The agreement between each trainee-assessors' INTER-NDA scores was compared with the expert's INTER-NDA scores, across INTER-NDA domains, for the four video recordings of the INTER-NDA described in the reliability experiment above. Protocol adherence scores were summed across all the items to yield an overall INTER-NDA protocol adherence score for each trainee-assessor, from which protocol adherence percentages (range 0-100\%) were calculated. The agreement between INTER-NDA domain scores, measured on the adapted INTER-NDA, was compared between trainee-assessors and the expert using kappa coefficients (Cohen, 1960, 1968).

\section{Statistical Analysis - Assessment of psychometric properties of the adapted INTER-NDA}

All data were analysed in SPSS Statistics v21.0.0.0 @ IBM Corp. Inter-rater and test-retest reliability between INTER-NDA scores across domains was determined for each trainee-assessor based on their scoring of four videos of the expert assessor administering the INTER-NDA to two-year old children. The second and fourth videos were identical, and trainee-assessors scored these videos separately, without access to the scores of video 1, allowing an assessment of test-retest reliability. Discussion between trainee-assessors and replaying of sections of the videos was not permitted. The video-based approach was selected over a conventional real-time approach to ensure that the child's scores were not affected by: (i) anxiety about performance in the presence a large number of assessors; and (ii) inability of assessors to hear and see the child's performance uniformly at all times during the assessment. This approach has been previously used in the INTERGROWTH-21st Project for reliability assessments (Fernandes et al., 2020; Murray et al., 2018). Each trainee-assessor administered the INTER-NDA on three children and internal consistency was determined for each INTER-NDA domain by calculating Cronbach's alphas (Bland \& Altman, 1997). Cronbach's alpha values are considered "good" if they were above a threshold of 0.7 (Tavakol \& Dennick, 2011). Test-retest and inter-rater reliability for the adapted INTER-NDA, across domains, was quantified using kappa coefficients (Cohen, 1960, 1968). Kappas of 0.81 and above are considered representative of almost perfect agreement, kappas of $0.61-0.80$ as substantial agreement, kappas of $0.41-0.60$ as moderate agreement, kappas of $0.21-0.40$ as fair agreement, and kappas of 0.20 and below as poor agreement (Cohen, 1968).

\section{Ethics}

The Institutional Review Board at St. George's University approved the study (IRB \#14066). All research personnel involved in the study completed the National Institutes of Health Office of Extramural Research Protecting Human Research Participants online course. Parents/guardians provided informed written consent on behalf of the participating children.

\section{Results}

\section{Adaptation of the INTER-NDA to the English-speaking Caribbean Context}

Following the evaluation of each of the 37 INTER-NDA items for linguistic and cultural relevance, eight items were identified to benefit from amendments to fit the local context. These were adapted to meet the study's requirements of cultural and linguistic prevalence, and the resultant amendments were scrutinized for conceptual equivalence with the 
corresponding original items of the INTER-NDA by the expert. The adaptations, as well the justifications for these, are presented in Table 3.

Table 3

\begin{tabular}{|ll|}
\hline Characteristic & N(\%) or Mean (SD) \\
\hline Sex & Female $-11(52.38 \%)$ \\
& Male $-10(47.62 \%)$ \\
\hline Ethnicity (Caribbean) & $21(100 \%)$ \\
\hline Native language (Caribbean-English) & $21(100 \%)$ \\
\hline Adaptation of the INTER-NDA Items to the Caribbean Context \\
\hline
\end{tabular}

\section{Psychometric properties of the adapted INTER-NDA}

The inter-rater and test-retest reliability of the INTER-NDA, across all domains, is presented in Table 4. Inter-rater reliability and test-retest reliability between the seven non-specialist child developmental assessors ranged between $\mathrm{K}=$ $0.99-1.00,95 \% \mathrm{Cl}[0.98,0.99]$ and $\mathrm{K}=0.76-1.00(95 \% \mathrm{Cl}[0.33,1.00])$ respectively, across all domains of the INTER-NDA, representing substantial to near-perfect agreement (Cohen, 1968). The Cronbach's alpha scores are presented in Table 5. These were good for the cognitive, language, gross motor, fine motor, and negative behavior domains $(a=0.89,0.84$, $1.00,0.75$, and 1.00 respectively) and sufficient for the positive behaviour domain $(a=0.64)$. The corresponding internal consistencies of the original INTER-NDA, previously published, are also presented in the table for comparison (Murray et al., 2018). 
Adaption meets criteria for:

\begin{tabular}{|c|c|c|c|c|c|c|c|}
\hline $\begin{array}{l}\text { INTER- } \\
\text { NDA } \\
\text { item } \\
\text { No. }\end{array}$ & $\begin{array}{l}\text { Original } \\
\text { INTER-NDA } \\
\text { item }\end{array}$ & $\begin{array}{l}\text { Adapted item } \\
\text { to the } \\
\text { Caribbean } \\
\text { context }\end{array}$ & $\begin{array}{l}\text { Rationale for } \\
\text { amendment }\end{array}$ & Justification & $\begin{array}{l}\text { Linguistic } \\
\text { relevance }\end{array}$ & $\begin{array}{l}\text { Cultural } \\
\text { relevance }\end{array}$ & $\begin{array}{l}\text { Conceptual } \\
\text { equivalence }\end{array}$ \\
\hline 1. & $\begin{array}{l}\text { Builds a tower } \\
\text { of } 5 \text { cubes } \\
\text { (Examiner } \\
\text { says "Could } \\
\text { you build a } \\
\text { tower with the } \\
\text { cubes?" }\end{array}$ & $\begin{array}{l}\text { Builds a tower } \\
\text { of } 5 \text { blocks } \\
\text { (Examiner } \\
\text { says "Could } \\
\text { you build a } \\
\text { tower with the } \\
\text { blocks?") }\end{array}$ & $\begin{array}{l}\text { Colloquialism } \\
\text { - 'Cubes' } \\
\text { replaced by } \\
\text { 'blocks' }\end{array}$ & $\begin{array}{l}\text { 'Cubes' are } \\
\text { referred to } \\
\text { as 'blocks' } \\
\text { in local } \\
\text { vernacular }\end{array}$ & $\checkmark$ & $\checkmark$ & $\checkmark$ \\
\hline
\end{tabular}

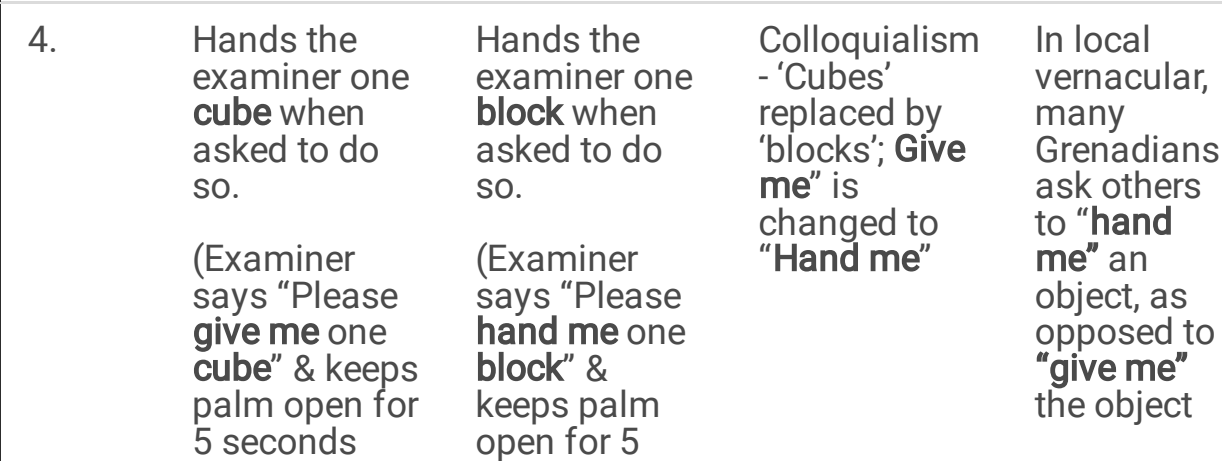

after child

has handed

seconds after

child has

over

handed over 1

1 cube)

block)"

8.

$\begin{array}{ll}\text { Points } & \text { Points } \\ \text { correctly } & \text { correctly } \\ \text { when asked } & \text { when asked } \\ \text { "Where is the } & \text { "Show me the } \\ \text { door/entrance } & \text { door/entrance } \\ \text { to the room?" } & \text { to the room?" }\end{array}$

Colloquialism Grenadians

- "Where is" is are often

changed to asked to

"Show me" "show"

where an

object is

located, as

opposed to

being asked

"where" the

object is

located

12. Able to make a cup of tea with the toy

tea set when requested by examiner

(Examiner

says "Can

you make a

cup of tea?")
Able to make a cup of tea with the toy kettle when requested by examiner (Examiner says "Can you make a cup of tea?")

Cultural
context - the
tea pot is
understood
to be a
"kettle" by the
children.

Many

Grenadians substitute a kettle for a teapot, as tea brewing is not a widely familiar practice on the island, especially among lowincome families. 


\begin{tabular}{|c|c|c|c|c|c|c|c|}
\hline \multirow[b]{2}{*}{14.} & \multirow[b]{2}{*}{$\begin{array}{l}\text { Feeds doll } \\
\text { when } \\
\text { requested to } \\
\text { (Examiner } \\
\text { says "Can } \\
\text { you give the } \\
\text { dolly some } \\
\text { tea?") }\end{array}$} & \multirow[b]{2}{*}{$\begin{array}{l}\text { Feeds doll } \\
\text { when } \\
\text { requested to } \\
\text { (Examiner } \\
\text { says "Can } \\
\text { you give the } \\
\text { dolly some } \\
\text { milk?") }\end{array}$} & \multirow[b]{2}{*}{$\begin{array}{l}\text { Cultural } \\
\text { context - tea } \\
\text { is understood } \\
\text { to be warm } \\
\text { milk or baby } \\
\text { formula. }\end{array}$} & \multicolumn{4}{|c|}{ Adaption meets criteria for: } \\
\hline & & & & $\begin{array}{l}\text { Grenadian } \\
\text { children are } \\
\text { not familiar } \\
\text { with the } \\
\text { concept of } \\
\text { tea, as the } \\
\text { term refers } \\
\text { to any hot } \\
\text { beverage- } \\
\text { not solely } \\
\text { tea as the } \\
\text { rest of the } \\
\text { world } \\
\text { knows it. } \\
\text { The age } \\
\text { group of } \\
\text { children } \\
\text { tested in the } \\
\text { study know } \\
\text { "tea" to be } \\
\text { warm milk. }\end{array}$ & $\checkmark$ & $\checkmark$ & $\checkmark$ \\
\hline 20. & $\begin{array}{l}\text { Throws a ball } \\
\text { very near } \\
\text { (Examiner } \\
\text { says "Can } \\
\text { you throw the } \\
\text { ball?" }\end{array}$ & $\begin{array}{l}\text { Throws a ball } \\
\text { very near } \\
\text { (Examiner } \\
\text { says "Can } \\
\text { you pelt the } \\
\text { ball?" or "Pelt } \\
\text { the ball for } \\
\text { me" or "Send } \\
\text { the ball for } \\
\text { me") }\end{array}$ & $\begin{array}{l}\text { Colloquialism } \\
\text { - "throw" is } \\
\text { changed to } \\
\text { "pelt" or } \\
\text { "send" }\end{array}$ & $\begin{array}{l}\text { When asked } \\
\text { using the } \\
\text { former } \\
\text { phrase, } \\
\text { Grenadian } \\
\text { children } \\
\text { would } \\
\text { gently drop } \\
\text { the ball in } \\
\text { front of } \\
\text { them. } \\
\text { Children } \\
\text { would } \\
\text { respond to } \\
\text { "pelt the } \\
\text { ball" by } \\
\text { throwing the } \\
\text { ball } \\
\text { overhand. }\end{array}$ & $\checkmark$ & $\checkmark$ & $\checkmark$ \\
\hline 26. & $\begin{array}{l}\text { Use of a } \\
\text { pronoun e.g. } \\
\text { [mine] me, my, } \\
\text { she, he, it, I } \\
\text { (Examiner } \\
\text { says "Who } \\
\text { does this } \\
\text { belong to? } \\
\text { "Whose is } \\
\text { this?") }\end{array}$ & $\begin{array}{l}\text { Use of a } \\
\text { pronoun e.g. } \\
\text { [mines] me, } \\
\text { my, she, he, it, } \\
\text { I } \\
\text { (Examiner } \\
\text { says "Who } \\
\text { dat/dis for?") }\end{array}$ & $\begin{array}{l}\text { Colloquialism } \\
\text { - "mine" is } \\
\text { changed to } \\
\text { "mines" and } \\
\text { "Whose is } \\
\text { this?" } \\
\text { changed to } \\
\text { "Who dat/dis } \\
\text { for?" }\end{array}$ & $\begin{array}{l}\text { Many } \\
\text { Grenadians } \\
\text { utilize "dat" } \\
\text { and/or "dis" } \\
\text { in } \\
\text { accordance } \\
\text { with local } \\
\text { vernacular. } \\
\text { Additionally, } \\
\text { phonetic } \\
\text { differences } \\
\text { between } \\
\text { singular and } \\
\text { plural words } \\
\text { is not } \\
\text { always } \\
\text { observed in } \\
\text { local } \\
\text { Grenadian } \\
\text { vernacular. }\end{array}$ & $\checkmark$ & $\checkmark$ & $\checkmark$ \\
\hline
\end{tabular}




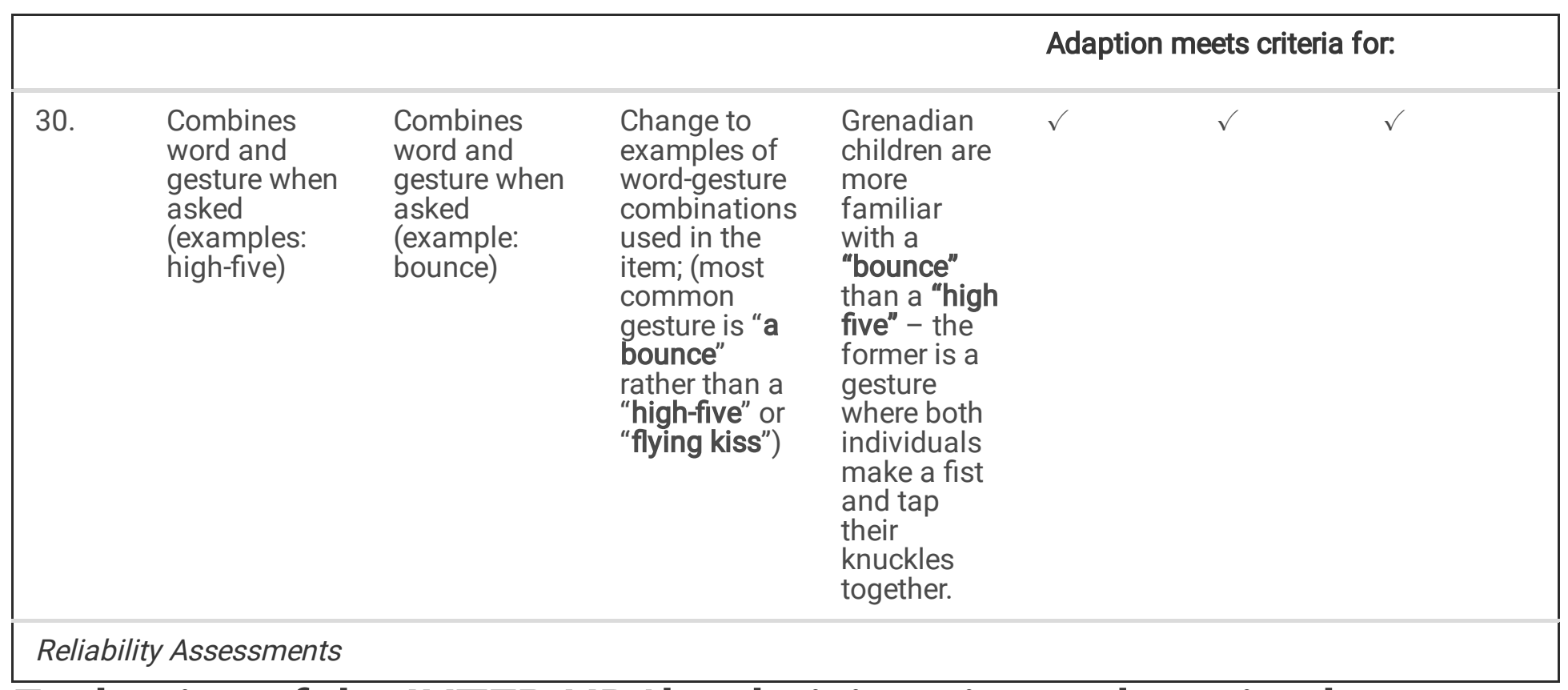

\section{Evaluation of the INTER-NDA's administration and scoring by non- specialist child development assessors}

The protocol adherence scores of non-specialist child development assessors in the context of the administration of the adapted INTER-NDA ranged between $70.5 \%$ and $100 \%(\mathrm{n}=7, M=89.9 \%, S D=10.1 \%)$ (Table 6).

Agreement between the expert and the non-specialist child developmental assessors is presented in Table 4 and ranged between $\mathrm{k}=0.89$ and $\mathrm{k}=1.00(95 \% \mathrm{Cl} 0.58,1.00)$ representing almost perfect agreement (Cohen, 1968).

Table 6 Protocol Adherence Scores fornon-specialist child developmental assessors for the adapted INTER-NDA

\begin{tabular}{|ll|}
\hline Assessor Number & \% Adherence \\
\hline 1 & 94.1 \\
\hline 2 & 82.4 \\
3 & 94.4 \\
\hline 4 & 94.1 \\
\hline 5 & 100 \\
\hline 6 & 94.1 \\
\hline 7 & 70.5 \\
\hline
\end{tabular}

\section{Discussion}

This study presents, to our knowledge, the first adaptation of the INTER-NDA to the linguistic and cultural context of the Caribbean, making the INTER-NDA the first ECD measure specifically adapted for use in the English-speaking Caribbean. The adapted INTER-NDA is very similar to the original INTER-NDA, with linguistic and cultural adaptations in 8 of the 37 items while simultaneously maintaining conceptual equivalence between the original and adapted versions. The interrater and test-retest reliability of the adapted INTER-NDA, and its internal consistency, were satisfactory across domains and comparable to the original INTER-NDA. Importantly, especially in the context of LMIC settings, we also showed that 
the adapted INTER-NDA can be administered and scored by non-specialist child development assessors at high levels of protocol adherence and agreement with an expert specialist assessor.

The results of our study provide evidence to support the use of the adapted INTER-NDA to measure ECD prevalence in the English-speaking Caribbean and for non-specialist child development assessors, trained and standardized in the INTER-NDA, to use the tool to conduct ECD evaluations. Previous studies have shown that the INTER-NDA can be administered in school settings in Mexico, and in research settings in Brazil, India, Italy, Kenya and the UK, by nonspecialist assessors at high levels of reliability and protocol adherence (Fernandes et al., 2020; Slim et al., 2018). These studies have focused on the translation, back translation and cultural adaptation of the INTER-NDA, developed initially in British English, into non-English languages. Our study extends this work by cultural and linguistically adapting the INTER-NDA for use in an English-speaking, yet culturally and geographically diverse, LMIC population, i.e. the Englishspeaking Caribbean. Moreover, in our study, we have presented a methodological template for this process, which we hope can be applied to the adaptation of other ECD tools for use in the Caribbean setting.

\section{Strengths and Limitations of the Study}

Our study is important in a number of ways. First, consideration was given to different lexical items to express similar concepts in British and Creole English; for example, "throw" and "pelt"; "cubes" and "blocks"; and "teapot" and "kettle. This is particularly important in the Grenadian context where children are more familiar with Creole English, rather than British English, during early life. Second, care was taken to ensure that the components of the INTER-NDA's kit were familiar to Caribbean children, and commonly encountered in Caribbean households. This is important because the use of items unfamiliar to the average child's repertoire of household and play-related exposures, such as a puffed rice grain or a maize bean (Khan et al., 2013; Gladstone et al., 2010); or items which the child may be forbidden from playing with, such as a matchbox (Gladstone et al., 2010), are important, yet often under-estimated, confounders of ECD assessments. Third, we assessed whether non-specialist child development assessors, in the Caribbean context, can measure ECD outcomes on the adapted INTER-NDA. This is an important consideration in LMIC settings where reliance, for ECD assessments on specialist professionals for their administration, scoring and interpretation is one of the key rate-limiting steps to the scalability of ECD surveillance and for the identification of children at risk who may benefit from interventions. Although non-specialists have been previously shown to administer and score the INTER-NDA reliably, this study is, to our knowledge, the first effort to assess this in a Caribbean setting and provides evidence to support ECD evaluations in Grenada by non-specialist child development assessors.

There are a number of limitations to consider. First, our sample size was small - we assessed the internal consistency and reliability of the adapted INTER-NDA, and its administration by non-specialists, across 7 trainee-assessors who performed 3 assessments each, i.e. a total of 21 assessments. Second, our study was carried out in the Grenadian setting and while socio-cultural similarities exist between the Caribbean nations, the piloting of the INTER-NDA on different Caribbean populations would be required before its wider use in these settings. Third, our adaptation of the INTER-NDA is limited to the English-speaking Caribbean, despite substantial proportions of the region's population being native in Spanish and French. Fourth, our results are restricted to the INTER-NDA's age range of assessment, i.e. the 22 to 30 month age group and, as such, ECD tools for the assessment of a wider age range of children are required to be adapted and standardized in order to significantly impact ECD surveillance in the Caribbean across the early childhood.

\section{Context of the Study}

An essential condition for achieving the United Nations Sustainable Development Goal (UN SDG) 4.2 ('ensure that all girls and boys have access to quality early child development, care and preprimary education so that they are ready for primary education') (United Nations, 2015) is the measurement of ECD outcomes at scale, in order to identify children at risk and to make cross-population comparisons (Fernandes et al., 2020). While a multitude of tools to measure ECD risk 
exist, none are culturally adapted and standardized for use in the Caribbean setting, especially for administration by nonspecialists. By linguistically and culturally adapting the INTER-NDA to the context of the English-speaking Caribbean, without compromising its conceptual integrity, we have, for the first time, produced a practically applicable, culturally relevant ECD measure specifically adapted to this setting. The INTER-NDA is a unique clinical tool for use across all healthcare systems to measure neurodevelopmental milestones and associated behaviors in 2-year-olds uniformly and at scale, and to identify children at risk of NDIs who would benefit from specialist referral and further investigation (Fernandes et al., 2020). It is our hope that the adapted INTER-NDA will complement the INTERGROWTH-21st Project's international INTER-NDA standards (Fernandes et al., 2020) for the measurement of ECD outcomes in the Caribbean while simultaneously providing a methodological template for the adaptation of child developmental measures to cultural and linguistic contexts.

\section{Conclusion}

This study represents, to our knowledge, the first adaptation of an ECD instrument to the English-speaking Caribbean. This is important for standardized, robust ECD measurement in the region, at scale, and supports the inclusion of Caribbean infants in international efforts at ECD surveillance. Furthermore, this study highlights the importance of the linguistic and cultural adaptation of ECD measures, even between settings which appear to share the same primary language but in which social, cultural, geographic and economic contexts vary.

\section{Declarations}

\section{Funding}

This study was supported by the Grand Challenges Canada Saving Brains Programme for the Grenada Saving Brains project, grant \# 0587-03. Michelle Fernandes is funded by the National Institute of Health Research, UK through a BRC Career Track fellowship in Paediatrics.

\section{Conflicts of Interest/Competing Interests}

None of the authors have a conflict of interest to declare.

\section{Availability of Data and Material}

The data that support the findings of this study are available from the corresponding author (RW) upon reasonable request.

\section{Ethics Approval}

The Institutional Review Board at St. George's University approved the study (IRB \#14066). All research personnel involved in the study completed the National Institutes of Health Office of Extramural Research Protecting Human Research Participants online course.

\section{Consent to Participate}

Parents/guardians provided informed written consent on behalf of the participating children.

\section{Consent for Publication}

All authors approved the final draft of the article and consent for the current study to be published. 
Acknowledgements

Not applicable.

Authors' Contributions

RW, MF, RE, TM, CM, SHo, RI, RWo, EA, and BL conducted the outlined methods. RW, RE, MF, and SH prepared the current manuscript.

\section{References}

1. Bakker C, Elings-Pels M, Reis M. (2009). The impact of migration on children in the Caribbean. UNICEF Office for Barbados \& Eastern Caribbean. Retrieved from https://www.unicef.org/easterncaribbean/Impact_of_Migration_Paper.pdf.

2. Bayley N. Bayley Scales of Infant and Toddler Development. 3rd ed. San Antonio: Harcourt Assessment; 2006.

3. Black MM, Lawn JE. Early childhood developmental disabilities-data still needed. The Lancet Global Health. 2018;6(10):e1050-1. doi:10.1016/s2214-109x(18)30399-1.

4. Bland JM, Altman DG. Statistics notes: Cronbach's alpha. BMJ. 1997;314(572):7080. http://dx.doi.org/10.1136/bmj.314.7080.572.

5. Central Intelligence Agency. (2018). Grenada. In The world factbook. Retrieved from https://www.cia.gov/library/publications/the-world-factbook/geos/gj.html.

6. Cohen J. A coefficient of agreement for nominal scales. Educ Psychol Measur. 1960;20(1):37-46.

7. Cohen J. Weighted kappa: Nominal scale agreement provision for scaled disagreement or partial credit. Psychol Bull. 1968;70(4):213-20. doi:10.1037/h0026256.

8. Cronbach LJ. Coefficient alpha and the internal structure of tests. Psychometrika. 1951;16(3):297-334. doi:10.1007/bf02310555.

9. Durkin M. The epidemiology of developmental disabilities in low-income countries. Mental Retardation Developmental Disabilities Research Reviews. 2002;8(3):206-11. doi:10.1002/mrdd.10039.

10. Farrar MJ, Ashwell S. Phonological awareness, executive functioning, and theory of mind. Cognitive Development. 2012;27(1):77-89. doi:10.1016/j.cogdev.2011.08.002.

11. Fernald LCH, Kariger P, Engle P, Raikes A. Examining early childhood development in low-income countries: A toolkit for the assessment of children in the first five years of life. Washington, DC: The World Bank; 2009. doi:10.1596/28107.

12. Fernandes M, Stein A, Newton CR, Cheikh-Ismail L, Kihara M, Wulff K,.. . Villar J. (2014). The INTERGROWTH-21st Project Neurodevelopment package: A novel method for the multi-dimensional assessment of neurodevelopment in pre-school age children. PLOS One, 9(11). doi:10.1371/journal.pone.0113360.

13. Fernandes M, Villar J, Stein A, Urias ES, Garza C, Victora CG, ... Kennedy S. (2020). INTERGROWTH-21st Project international INTER-NDA standards for child development at 2 years of age: An international prospective population-based study. BMJ Open, 10(6). doi:10.1136/bmjopen-2019-035258.

14. Gladkova A. Grammar and the influence of society and culture. The Encyclopedia of Applied Linguistics. 2012. doi:10.1002/9781405198431.wbeal0471.

15. Gladstone M, Lancaster GA, Umar E, Nyirenda M, Kayira E, Broek NR, Smyth RL. (2010). The Malawi Developmental Assessment Tool (MDAT): The creation, validation, and reliability of a tool to assess child development in rural African settings. PLOS Medicine, 7(5). doi:10.1371/journal.pmed.1000273.

Page $13 / 15$ 
16. Government of Grenada. (2005). Grenada national strategic plan for health, 2006-2010. Situational analysis. Health for economic growth and human development. Retrieved from http://www.gov.gd/egov/docs/other/nationalstrategic-health-plan-2006-2010.pdf.

17. Grantham-McGregor S, Cheung YB, Cueto S, Glewwe P, Richter L, Strupp B. Developmental potential in the first 5 years for children in developing countries. The Lancet. 2007;369(9555):60-70. doi:10.1016/s0140-6736(07)600324.

18. Khan NZ, Muslima H, Shilpi AB, Begum D, Parveen M, Akter N,.. . Darmstadt GL. (2013). Validation of rapid neurodevelopmental assessment for 2- to 5-year-old children in Bangladesh. Pediatrics, 131(2). doi:10.1542/peds.2011-2421.

19. Lambert MC, Sewell WC, Levitch AH. Metamorphosing Euro American psychological assessment instruments to measures developed by and for English-speaking Caribbean people. In: Roopnarine JL, Chadee D, editors. Caribbean psychology: Indigenous contributions to a global discipline. Washington, DC, US: American Psychological Association; 2016. pp. 327-55. doi:10.1037/14753-014.

20. Lambert MC, Williams SG, Morrison JW, Samms-Vaughan ME, Mayfield WA, Thornburg KR. Are the indicators for the language and reasoning subscale of the early childhood environment rating scale-revised psychometrically appropriate for Caribbean classrooms? International Journal of Early Years Education. 2008;16(1):41-60. doi:10.1080/09669760801892219.

21. Landon B, Waechter R. Reducing Corporal Punishment with Saving Brains Grenada. Presented at the International Congress on Psychology. Japan: Yokohama; 2016.

22. Lu C, Black MM, Richter LM. Risk of poor development in young children in low-income and middle-income countries: An estimation and analysis at the global, regional, and country level. The Lancet Global Health. 2016;4(12):e916-22. doi:10.1016/s2214-109x(16)30266-2.

23. Murray E, Fernandes M, Newton CR, Abubakar A, Kennedy SH, Villar J, Stein A. (2018). Evaluation of the INTERGROWTH-21st Neurodevelopment Assessment (INTER-NDA) in 2 year-old children. PLOS One, 13(2). doi:10.1371/journal.pone.0193406.

24. Olusanya BO, Davis AC, Wertlieb D, Boo N, Nair M, Halpern R,.. . Kassebaum NJ. Developmental disabilities among children younger than 5 years in 195 countries and territories, 1990-2016: A systematic analysis for the global burden of disease study 2016. The Lancet Global Health. 2018;6(10):e1100-21. doi:10.1016/s2214$109 \times(18) 30309-7$.

25. Patterson JL. Comparing bilingual and monolingual toddlers expressive vocabulary size. Journal of Speech Language Hearing Research. 2004;47(5):1213-5. doi:10.1044/1092-4388(2004/089).

26. Razzouk D, Gallo C, Olifson S, Zorzetto R, Fiestas F, Poletti G,.. . Mari JJ. Challenges to reduce the '10/90 gap': Mental health research in Latin American and Caribbean countries. Acta Psychiatr Scand. 2008;118(6):490-8. doi:10.1111/j.1600-0447.2008.01242.x.

27. Rothbart MK. Measurement of temperament in Infancy. Child Dev. 1981;52(2):569-78. doi:10.2307/1129176.

28. Samms-Vaughn M. Comprehensive longitudinal studies of child health, development and behavior in Jamaica: Findings and policy impact. West Indian Med J. 2008;57(6):639-44.

29. Semrud-Clikeman M, Romero RAA, Prado EL, Shapiro EG, Bangirana P, John CC. Selecting measures for the neurodevelopmental assessment of children in low- and middle-income countries. Child Neuropsychol. 2016;23(7):761-802. https://doi.org/10.1080/09297049.2016.1216536.

30. Slim J, Villar J, Varela GG, Castillo FH, Duran AB, Guzman MDLL, ... Fernandes M. Individual or group-based approach to the assessment of preschool children: A comparison using the INTERGROWTH-21st 
Neurodevelopmental Assessment (INTER-NDA). International Journal of Growth Development. 2018;2:11-33. doi:10.25081/ijgd.2018.v2.60.

31. Tavakol M, Dennick R. (2011). Making sense of Cronbach's alpha. International Journal of Medical Education, 2(53). https://doi.org/10.5116/ijme.4dfb.8dfd.

32. United Nations, Department of Economic and Social Affairs. (2015). Transforming our world: The 2030 agenda for sustainable development. Retrieved from https://sdgs.un.org/publications/transforming-our-world-2030-agendasustainable-development-17981.

33. United Nations, Department of Economic and Social Affairs, Population Division. (2017). World population prospects: The 2017 revision. Retrieved from https://population.un.org/wpp/DataQuery/.

34. Villar J, Altman DG, Purwar M, Noble JA, Knight HE, Ruyan P, ... Kennedy SH. The objectives, design and implementation of the INTERGROWTH-21st Project. BJOG. 2013;120(Suppl 2):9-26. doi:10.1111/14710528.12047.

35. World Bank. (2016). GNI per Capita, PPP (current international \$). Retrieved from http://data.worldbank.org/indicator/NY.GNP.PCAP.PP .CD? order=wbapi_data_value_2014+wbapi_data_value+wbapi_data_value-last\&sort=asc.

36. World Bank. (2018). World Bank country and lending groups. Retrieved from https://datahelpdesk.worldbank.org/knowledgebase/articles/906519.

\section{Supplementary Files}

This is a list of supplementary files associated with this preprint. Click to download.

- floatimage1.png

- floatimage2.png

- floatimage3.png

- floatimage4.png

- floatimage5.png

- floatimage6.jpeg 